\title{
NUMERICAL SIMULATION OF TEMPERATURE-DRIVEN FREE SURFACE FLOWS, WITH APPLICATION TO LASER SURFACE MELTING
}

\author{
A. CABOUSSAT ${ }^{1}$, J. HESS ${ }^{2}$, A. MASSEREY ${ }^{3}$, AND M. PICASSO ${ }^{4}$ \\ ${ }^{1}$ Geneva School of Business Administration, University of Applied Sciences and Arts Western \\ Switzerland (HES-SO), alexandre.caboussat@ hesge.ch, http://campus.hesge.ch/caboussat \\ ${ }^{2}$ Geneva School of Business Administration, University of Applied Sciences and Arts Western \\ Switzerland (HES-SO), and Ycoor Systems SA, 3960 Sierre, Switzerland, julien.hess@ @esge.ch, \\ julien.hess@ycoorsystems.com \\ ${ }^{3}$ Ycoor Systems SA, 3960 Sierre, Switzerland, masserey@ycoorsystems.com \\ ${ }^{4}$ Institute of Mathematics, Ecole polytechnique fédérale de Lausanne, 1015 Lausanne, Switzerland, \\ marco.picasso@epfl.ch
}

Key words: Navier-Stokes equations, solidification, operator splitting, two-grid, surface tension, Marangoni effects, shallow laser surface melting

Abstract. A mathematical model coupling the heat and fluid flow with solidification and free surfaces is presented. The numerical method relies on an operator splitting strategy, and a two-grid method. The free surfaces are tracked with a volume-of-fluid approach. A special emphasis is laid on the modeling of surface tension forces on the free surface. A comparison between approaches is highlighted, and a mesh convergence analysis is presented. Finally, the model is validated with the simulation of a static laser melting process.

\section{INTRODUCTION}

In shallow laser surface melting (SLSM) processes, the heat source produced by a laser source allows to (re)melt solid metal locally in order to obtain a polished surface after re-solidification. Such industrial processes are well-known in engineering, see, e.g., [1,2], for instance on surfaces obtained by additive manufacturing [3, 4].

The simulation of this kind of industrial processes requires a coupled mathematical model that incorporates the flow effects, through the incompressible Navier-Stokes equations, the heat effects, through an advection-diffusion heat equation, and the tracking of the free surfaces. When melting, velocity currents are created in the metal melt pool, due to surface tension effects. In particular, temperature gradients introduce a tangential force on the free surface that creates the internal flow motion, and that will eventually lead to the time-evolution and deformation of the metal-air interface.

In this work, we focus in particular on two different numerical approaches for the modeling of surface forces. The first approach relies on an explicit calculation of the free surface curvature based on [5], 
while the second approach relies on an implicit calculation of the surface force, and includes contact angles with the domain boundary if needed, as in [6]. Following [5, 7], the numerical method relies on an operator splitting algorithm, and a two-grid method. The convergence of the approximation of the solution when the discretization parameters tend to zero is evaluated through the behavior of the deformed free surface, and numerical results show appropriate first order convergence.

\section{MATHEMATICAL MODEL}

Let us consider a bounded domain $\Lambda \subset \mathbb{R}^{3}$, and let $T>0$ be the final time of the simulation. For any given time $t \in(0, T)$, let $\Omega_{t} \subset \Lambda$ be the domain occupied by the metal (solid or not) and $\Gamma_{t}:=\partial \Omega_{t} \backslash \partial \Lambda$ be the free surface between the metal and the ambient air. Figure 1 typically illustrates a $2 \mathrm{D}$ setup for the laser melting of a thin metal plate.

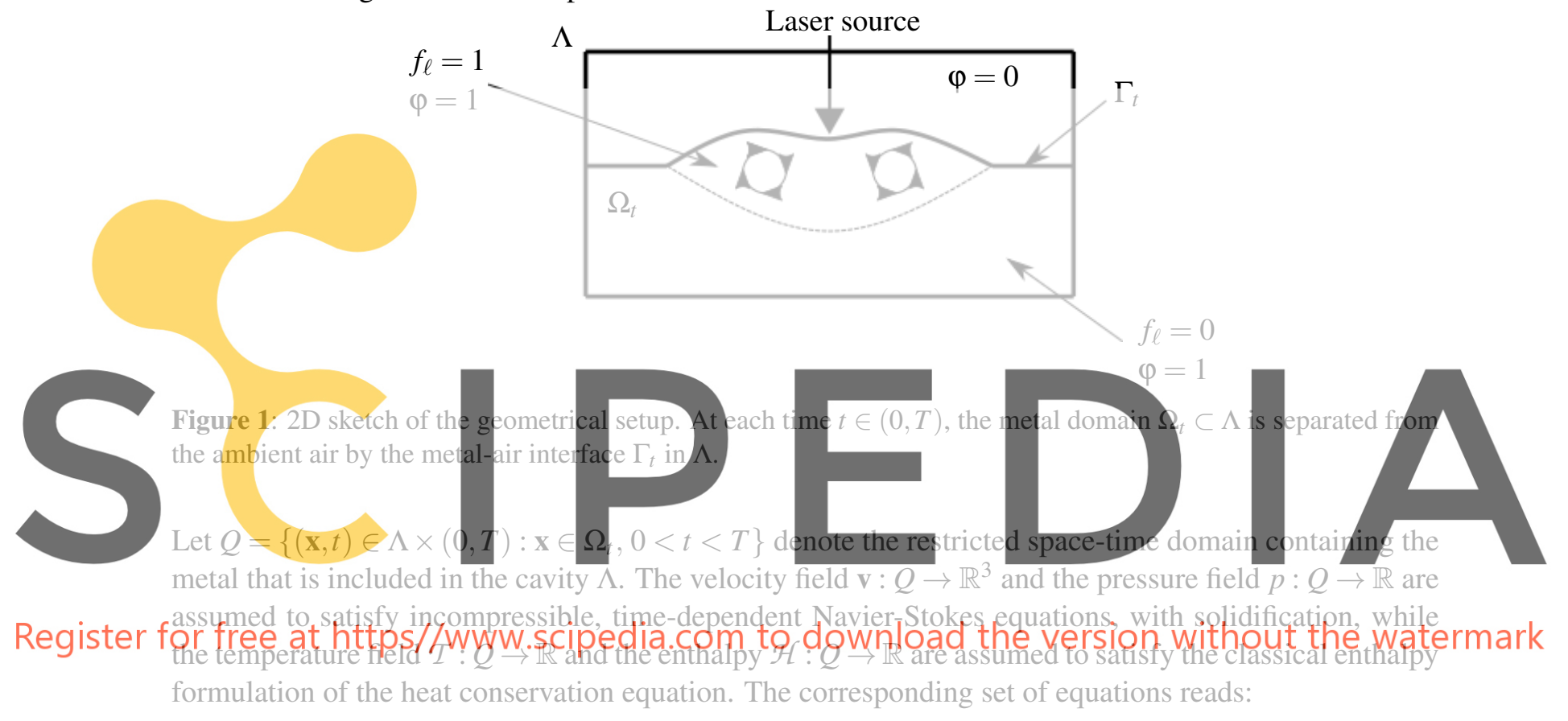

$$
\begin{aligned}
& \rho \frac{\partial \mathbf{v}}{\partial t}+\rho(\mathbf{v} \cdot \nabla) \mathbf{v}-2 \nabla \cdot(\mu \mathbf{D}(\mathbf{v}))+\alpha(\mathcal{T}) \mathbf{v}+\nabla p=\rho \mathbf{g}, \\
& \nabla \cdot \mathbf{v}=0 \\
& \frac{\partial \mathcal{H}}{\partial t}+\mathbf{v} \cdot \nabla \mathcal{H}-\nabla \cdot(k \nabla \mathcal{T})=0 .
\end{aligned}
$$

Here $\mathbf{D}(\mathbf{v})=\frac{1}{2}\left(\nabla \mathbf{v}+\nabla \mathbf{v}^{T}\right)$ is the symmetric deformation tensor, $\rho$ and $\mu$ are respectively the density and the viscosity of the metal and $\mathbf{g}$ denotes the gravity acceleration. Here $k$ is the thermal conductivity of the metal, and $\mathcal{T}=\beta(\mathcal{H})$ is the inverse relationship of the monotonic enthalpy function

$$
\mathcal{H}(\mathcal{T})=\int_{0}^{\mathcal{T}} \rho C_{p}(s) d s+L f_{\ell}(\mathcal{T})
$$


where $C_{p}$ is the specific heat, $L$ is the latent heat, and $f_{\ell}=f_{\ell}(\mathcal{T})$ is the liquid fraction, which equals one in the liquid region (above the temperature of fusion) and zero in the solid region. In order to model solidification [8], the reaction coefficient in (1) is given by:

$$
\alpha(\mathcal{T})=\bar{\alpha} \frac{\mu\left(1-f_{\ell}(\mathcal{T})\right)^{2}}{\left(f_{\ell}(\mathcal{T})+\varepsilon\right)^{3}}
$$

where $\bar{\alpha}$ is a constant, and $0<\varepsilon \ll 1$.

Let $\varphi: \Lambda \times(0, T) \rightarrow\{0,1\}$ be the characteristic function of the metal domain, which equals one if metal is present and zero otherwise. The space-time metal domain can be equivalently defined as $Q=$ $\{(\mathbf{x}, t) \in \Lambda \times(0, T): \varphi(\mathbf{x}, t)=1\}$. The time evolution of the metal domain $\Omega_{t} \subset \Lambda$ is modeled by means of a volume-of-fluid method. In order to describe the kinematics of the free surface, the characteristic function $\varphi$ must satisfy (in a weak sense):

$$
\frac{\partial \varphi}{\partial t}+\mathbf{v} \cdot \nabla \varphi=0 \quad \text { in } \Lambda \times(0, T)
$$

where $\mathbf{v}$ in $\Lambda \times(0, T) \backslash Q$ is a regular extension of $\mathbf{v}$ outside $Q$, see, e.g., [7]. The model is completed with appropriate initial and boundary conditions. In particular, surface tension and Marangoni effects on the metal-air interface are taken into account via a force term on the free surface $\Gamma_{t}[9,10]$. The ambient air is assumed to have no influence on the metal, and is treated as vacuum. The boundary conditions on the metal-air interface $\Gamma_{t}=\partial \Omega_{t} \backslash \partial \Lambda$ are thus given by:
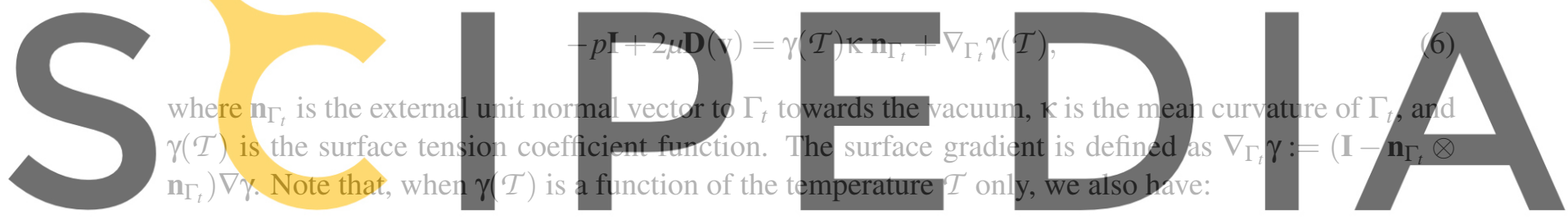

$\nabla_{\Gamma_{t}} \gamma(\mathcal{T})=\gamma^{\prime}(\mathcal{T}) \nabla \mathcal{T} \cdot \mathfrak{t}_{\Gamma_{t}, 1}+\gamma^{\prime}(\mathcal{T}) \nabla \mathcal{T} \cdot \mathfrak{t}_{\Gamma_{t}, 2}$

(7)

Register for free at https//www.scipedia.com to download the version without the watermark where $t_{\Gamma_{t}, i}, i=1,2$, are two linearly independent vectors in the tangent plane to $\Gamma_{t}$, perpendicular to $\mathbf{n}_{\Gamma_{t}}$.

Finally, the laser source is modeled via a prescribed heat flux on the boundary $\Gamma_{t}$ of the metal domain, while adiabatic boundary conditions are typically applied on the rest of the boundary. More precisely, the laser source condition is written as $k \frac{\partial \mathcal{T}}{\partial \mathbf{n}_{\Gamma_{t}}}=g_{f}$ on $\Gamma_{t}$, where $g_{f}$ is a given heat flux.

\section{TIME DISCRETIZATION}

The implicit splitting algorithm of order one described in [5, 7] for Newtonian flows is extended here to temperature-dependent free surface flows. It relies on operator splitting and on a two-grid method: the splitting algorithm decouples advection and diffusion phenomena, while the two-grid method allows to increase the accuracy of the approximation of the free surface by considering a finer grid for the approximation of the advection problems.

Let $0=t^{0}<t^{1}<t^{2}<\ldots<t^{N}=T$ be a subdivision of the time interval $[0, T]$ and $\tau^{n}:=t^{n+1}-t^{n}$ be the $(n+1)$ th time step, $n=0,1,2, \ldots, N-1$. The operator splitting algorithm is described as follows, and illustrated in Figure 2. 


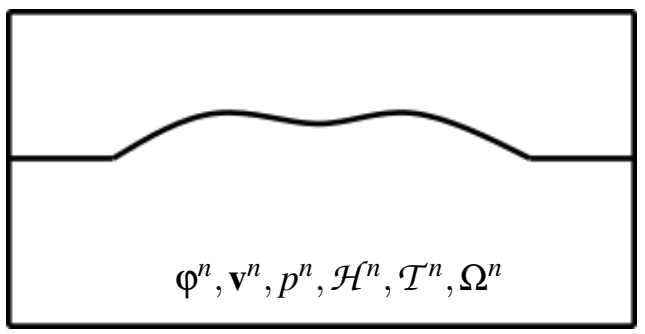

Time $t^{n}$

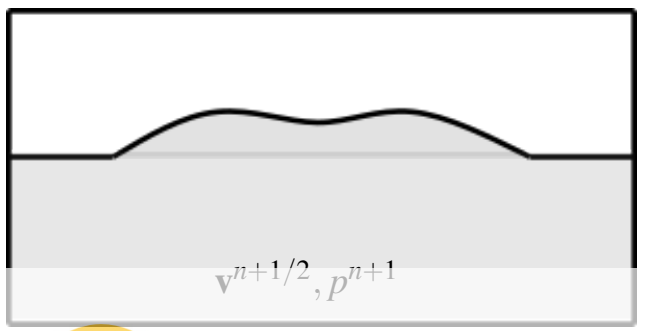

(2) Stokes problem

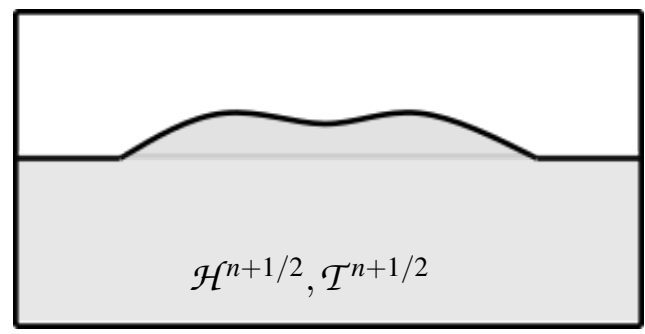

(1) Thermal step

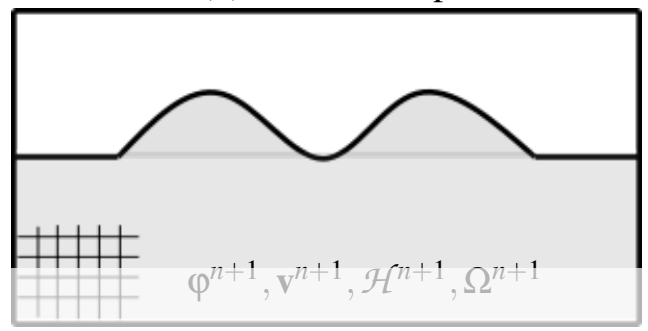

(3) Advection step

Figure 2: Operator splitting algorithm (from top to bottom). At each time step $n$, we first solve the heat equation (without convection) to determine $\mathcal{H}^{n+1 / 2}$ and $\mathcal{T}^{n+1 / 2}$. Then, a generalized Stokes problem is solved in order to obtain $\mathbf{v}^{n+1 / 2}$ and $p^{n+1}$. Finally, three advection problems are solved in order to obtain $\varphi^{n+1}$ (and thus $\Omega^{n+1}$ ), $\mathbf{v}^{n+1}$ and $\mathcal{H}^{n+1}$
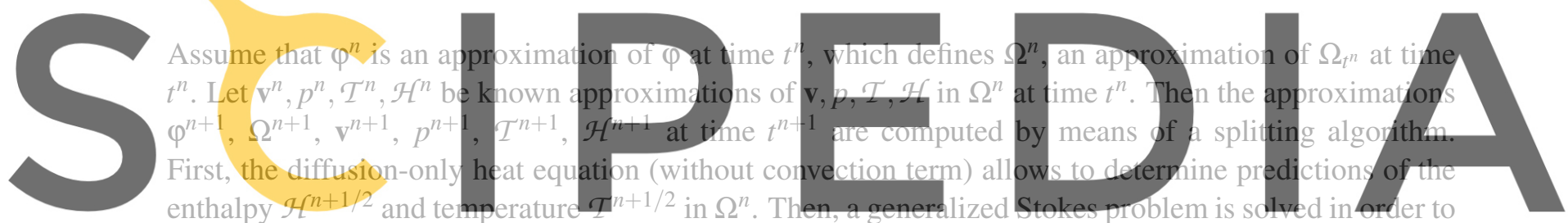

obtain the predicted velocity $\mathbf{v}^{n+1 / 2}$ and the pressure $p^{n+1}$ in $\Omega^{n}$. Finally, advection problems are solved

Register föroficeet enthalpy $\mathcal{H}^{n+1}$ (and thus the corrected temperature $\mathcal{T}^{n+1}=\beta\left(\mathcal{H}^{n+1}\right)$ ) in $\Omega^{n+1}$.

\subsection{Heat equation}

The domain $\Omega^{n}$ being given, we consider the problem: find $\mathcal{H}: \Omega^{n} \rightarrow \mathbb{R}$ and $\mathcal{T}: \Omega^{n} \rightarrow \mathbb{R}$ satisfying

$$
\frac{\partial \mathcal{H}}{\partial t}-\nabla \cdot(k \nabla \mathcal{T})=0 \quad \text { in } \Omega^{n}
$$

with appropriate boundary conditions on the boundary $\partial \Omega^{n}$. We use an implicit Euler scheme for its time-discretization:

$$
\frac{\mathcal{H}^{n+1 / 2}-\mathcal{H}^{n}}{\tau^{n}}-\nabla \cdot\left(k \nabla \mathcal{T}^{n+1 / 2}\right)=0 \quad \text { in } \Omega^{n},
$$

where $\mathcal{T}^{n+1 / 2}=\beta\left(\mathcal{H}^{n+1 / 2}\right)$. This time-discretized system of equations is solved with the so-called Chernoff numerical scheme $[11,12]$. Once the temperature $\mathcal{T}^{n+1 / 2}$ is known, one can determine $f_{\ell}\left(\mathcal{T}^{n+1 / 2}\right)$, and $\alpha^{n+1 / 2}=\alpha\left(\mathcal{T}^{n+1 / 2}\right)$. 


\subsection{Diffusion operator}

Then, a generalized Stokes problem is solved in order to obtain the predicted velocity and the pressure in the metal domain $\Omega^{n}$. We consider the problem: find $\mathbf{v}: \Omega^{n} \rightarrow \mathbb{R}^{3}$ and $p: \Omega^{n} \rightarrow \mathbb{R}$ satisfying (together with the natural force condition on the metal-air interface, as described below):

$$
\begin{aligned}
& \rho \frac{\partial \mathbf{v}}{\partial t}-2 \nabla \cdot(\mu \mathbf{D}(\mathbf{v}))+\alpha^{n+1 / 2} \mathbf{v}+\nabla p=\rho \mathbf{g} \\
& \nabla \cdot \mathbf{v}=0 .
\end{aligned}
$$

An implicit Euler scheme is used for the time discretization of this Stokes system in $\Omega^{n}$ :

$$
\begin{aligned}
& \rho \frac{\mathbf{v}^{n+1 / 2}-\mathbf{v}^{n}}{\tau^{n}}-2 \nabla \cdot\left(\mu \mathbf{D}\left(\mathbf{v}^{n+1 / 2}\right)\right)+\alpha^{n+1 / 2} \mathbf{v}^{n+1 / 2}+\nabla p^{n+1}=\rho \mathbf{g}, \\
& \nabla \cdot \mathrm{v}^{n+1 / 2}=0 .
\end{aligned}
$$

with no-slip or pure-slip conditions on the boundary of the cavity $\partial \Lambda$ and the natural force condition on the metal-air interface:

$-p^{n+1} \mathbf{I}+2 \mu \boldsymbol{D}\left(\mathbf{v}^{n+1 / 2}\right)=\gamma\left(\mathcal{T}^{n+1 / 2}\right) \boldsymbol{K}^{n} \mathbf{n}_{\Gamma^{n}}+\nabla_{\Gamma^{n}} \gamma\left(\mathcal{T}^{n+1 / 2}\right)$

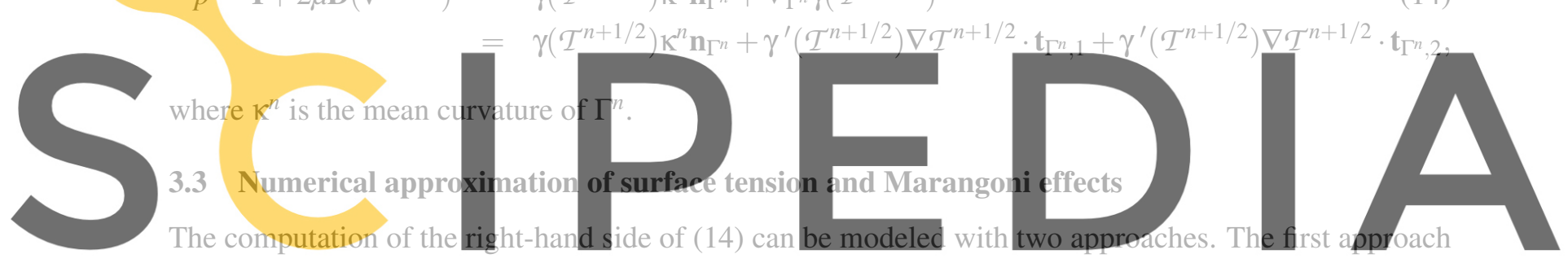

relies on an explicit computation of the mean curvature $\kappa^{n}$ through a convolution smoothing of $\varphi^{n}$, see,

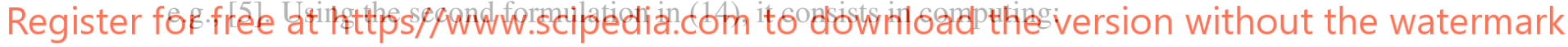

$$
\nabla \tilde{\varphi}^{n}(\mathbf{x})=\int_{\Lambda} \varphi^{n}(\mathbf{y}) \nabla K_{\varepsilon}(\mathbf{x}-\mathbf{y}) \mathrm{d} \mathbf{y}, \quad \forall \mathbf{x} \in \Lambda,
$$

where $K_{\mathcal{\varepsilon}}(\cdot)$ is a smoothing kernel with radius $\varepsilon$. Then the curvature is defined by $\kappa^{n}:=-\nabla \cdot\left(\frac{\nabla \tilde{\varphi}^{n}}{\left\|\nabla \tilde{\varphi}^{n}\right\|}\right)$, and the tangential terms are explicitly calculated.

The second approach relies on a variational approach introduced in [6]. Using the first formulation of (14), it consists in transforming, in the weak formulation, the right-hand side of (14) as follows:

$$
\begin{aligned}
\int_{\Gamma^{n}}\left[\gamma\left(\mathcal{T}^{n+1 / 2}\right) \kappa^{n} \mathbf{n}_{\Gamma^{n}}+\nabla_{\Gamma^{n}} \gamma\left(\mathcal{T}^{n+1 / 2}\right)\right] \cdot \mathbf{w} d S= & -\int_{\Gamma^{n}} \gamma\left(\mathcal{T}^{n+1 / 2}\right) \operatorname{tr}\left(\nabla_{\Gamma^{n}} \mathbf{w}\right) d S \\
& +\int_{\partial \Gamma^{n}} \gamma\left(\mathcal{T}^{n+1 / 2}\right) \cos \left(\theta_{S}\right) \mathbf{t}_{\partial \Omega} \cdot \mathbf{w} d \ell
\end{aligned}
$$

where $\theta_{s}$ is the static contact angle and $\mathbf{t}_{\partial \Omega}$ is defined as in [6], and $\mathbf{w}$ is a test function. Actually, 
following [6], we can check that (16) relies on the following relationship:

$$
\begin{aligned}
\int_{\Gamma} \kappa(\gamma \mathbf{w}) \cdot \mathbf{n}_{\Gamma} d S & =-\int_{\Gamma} \operatorname{tr}\left(\nabla_{\Gamma} \gamma \mathbf{w}\right) d S+\int_{\partial \Gamma} \gamma \cos \left(\theta_{s}\right) \mathbf{t}_{\partial \Omega} \cdot \mathbf{w} d \ell \\
& =-\int_{\Gamma} \gamma \operatorname{tr}\left(\nabla_{\Gamma} \mathbf{w}\right) d S-\int_{\Gamma} \operatorname{tr}\left(\nabla_{\Gamma} \gamma \otimes \mathbf{w}\right) d S+\int_{\partial \Gamma} \gamma \cos \left(\theta_{s}\right) \mathbf{t}_{\partial \Omega} \cdot \mathbf{w} d \ell \\
& =-\int_{\Gamma} \gamma \operatorname{tr}\left(\nabla_{\Gamma} \mathbf{w}\right) d S-\int_{\Gamma} \nabla_{\Gamma} \gamma \cdot \mathbf{w} d S+\int_{\partial \Gamma} \gamma \cos \left(\theta_{s}\right) \mathbf{t}_{\partial \Omega} \cdot \mathbf{w} d \ell .
\end{aligned}
$$

\subsection{Advection operator}

Finally, considering the advection operators in (1), (3) and (5), the last step consists in transporting $\varphi^{n}$, $\mathcal{H}^{n+1 / 2}$ and $\mathbf{v}^{n+1 / 2}$. It allows to obtain $\varphi^{n+1}$ (and thus $\Omega^{n+1}$ ), $\mathbf{v}^{n+1}$ and $\mathcal{H}^{n+1}$. It consists in solving, between $t^{n}$ and $t^{n+1}$ the following nonlinear equations:
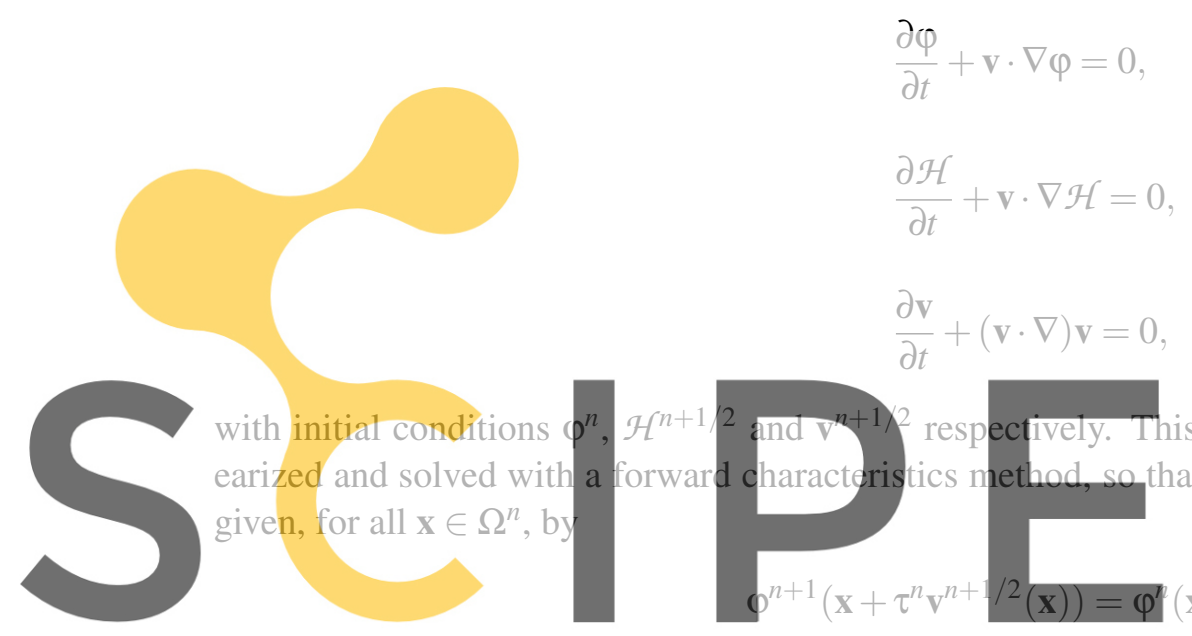

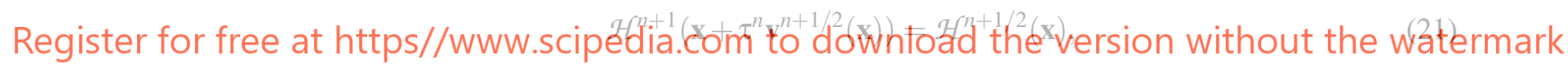

$$
\mathbf{v}^{n+1}\left(\mathbf{x}+\tau^{n} \mathbf{v}^{n+1 / 2}(\mathbf{x})\right)=\mathbf{v}^{n+1 / 2}(\mathbf{x}),
$$

\section{SPACE DISCRETIZATION}

In order to solve this multi-physics problem, we use a two-grid method approach as presented in [5, 7]. As illustrated in Figure 3 (in two dimensions), the space discretization of $\Lambda$ relies at each time step on a coarser unstructured tetrahedral finite element discretization $\mathcal{T}_{H}$ (with typical mesh size $H$ ), and a regular grid $C_{h}$ of smaller structured cells (with typical cell size $h$ ).

More precisely, the discretization $\mathcal{T}_{H}$ is restricted to the metal domain $\Omega^{n}$ at each time step to solve (9) and (12)-(13) with a finite elements method. The finite element mesh is adapted in the neighborhood of the free surface $\Gamma^{n}$, in order to have a better fit of the free surface, see, e.g., [13]. In particular, the resulting triangulated mesh of the free surface allows to compute the normal vector $\mathbf{n}_{\Gamma^{n}}$ as the normal vector to the triangular faces of that interface. On the other hand, the cavity $\Lambda$ is embedded into a parallelepiped box discretized into the structured Cartesian grid $\mathcal{C}_{h}$ made out of small parallelipipedic cells with a typical size $h$. 
The two-grid method allows to use the finer structured grid to increase the accuracy of the approximation of $\varphi^{n+1}$, and thus of the free surface $\Gamma^{n+1}$, by decreasing the numerical diffusion of the approximation $\varphi^{n+1}$ in (17), while maintaining reasonable computational costs of solving parabolic problems (such as the Stokes problem). We advocate $3 \leq H / h \leq 5$ for a reasonable trade-off [7]. Interpolation operators allow to transfer numerical approximations of the unknowns from $\mathcal{T}_{H}$ onto $C_{h}$ and vice-versa [14].
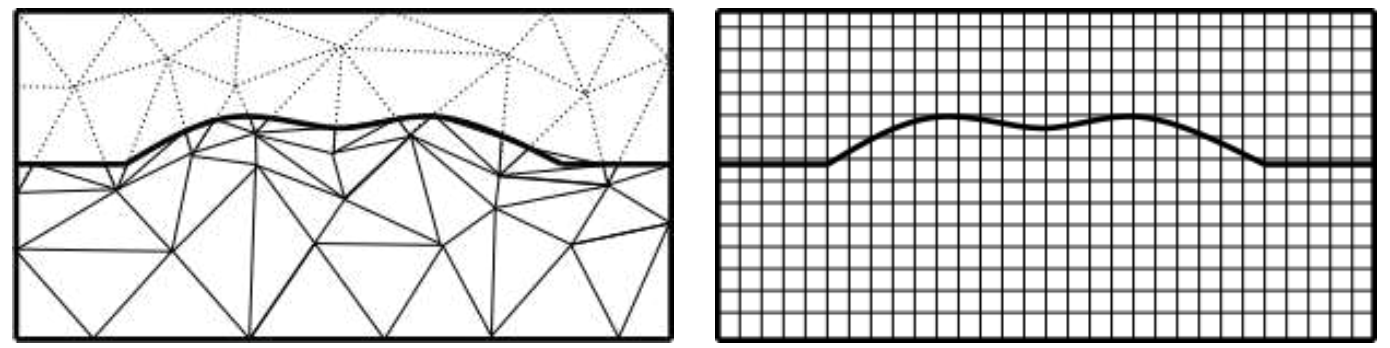

Figure 3: Two-grid method (2D sketch). The diffusion problems (heat equation and Stokes problem) are solved, in the metal domain, on an unstructured finite element mesh of typical size $H$, that is fitted to the free surface at reach time step (left). The advection problems are solved on a structured grid of small cubic cells of typical size $h$ (right).

One time step of the complete space-discretized first-order operator splitting algorithm may then be sketched as follows. Let us assume that piecewise constant approximations of $\varphi^{n}, \mathbf{v}^{n}$ and $\mathcal{H}^{n}$ are given

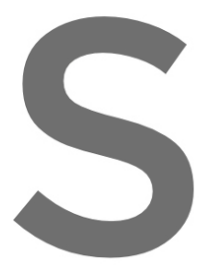
on the structured grid $C_{h}$. We proceed as follows:
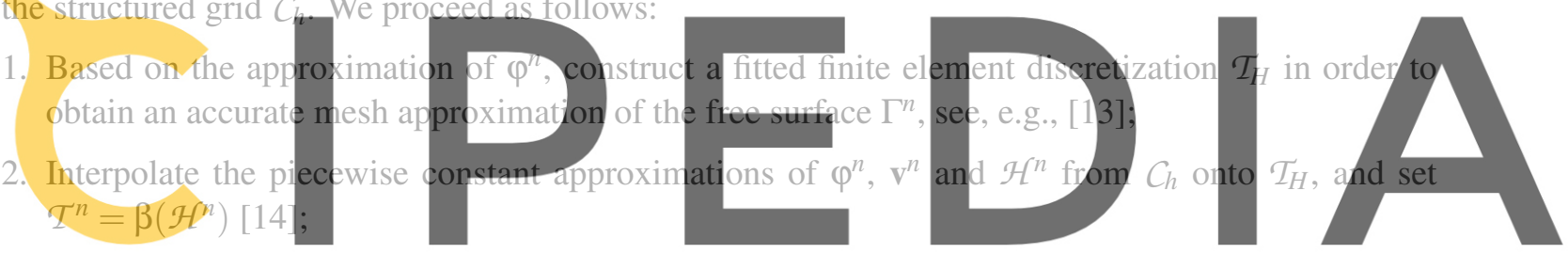

3. Solve the heat problem (9) with piecewise linear finite elements on $\mathcal{T}_{H}$ and a Chernoff numeri-

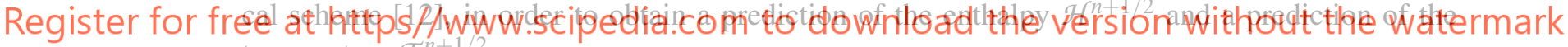
temperature $\mathcal{T}^{n+1 / 2}$.

4. Solve the Stokes problem (12)(13) with piecewise linear $\mathbb{P}_{1}$-bubble/ $\mathbb{P} 1$ finite elements $[15]$ on $\mathcal{T}_{H}$, in order to obtain a prediction of the velocity $\mathbf{v}^{n+1 / 2}$ and the pressure $p^{n+1}$.

5. Interpolate the piecewise linear approximations of $\mathbf{v}^{n+1 / 2}$ and $\mathcal{H}^{n+1 / 2}$ from $\mathcal{T}_{H}$ onto $\mathcal{C}_{h}$ [14];

6. Solve the advection problems $(20)(21)(22)$ on $\mathcal{C}_{h}$, in order to compute piecewise constant approximations of $\varphi^{n+1}$, and the corrections $\mathcal{H}^{n+1}, \mathbf{v}^{n+1}$ with a forward characteristics method.

\section{NUMERICAL EXPERIMENTS}

Simulation results are presented to validate the numerical methods with an experiment on static laser melting. Preliminary results can be found in [16], and are extended here for a thorough comparison of surface tension models.

This numerical experiment consists of a single static laser source melting a piece of metal [17] in a pseudo-2D configuration. It allows to validate the full coupling between flow and heat equations, with Marangoni effects on the free surface, and to study the convergence properties of the algorithm. 
The setup is that of Figure 1. We start the simulation with a flat free surface such that $\Omega_{0}=(-2.5,2.5) \times$ $(0, H) \times(4.6,7)[\mathrm{mm}]$, where $H=5 / N$ is the finite-element mesh size, for $N=24,48,96$ and 192. The structured Cartesian grid defined over $\Lambda$ is such that $h=H / 3[\mathrm{~mm}]$.

We consider the Böhler S705 steel as the material, whose physical properties are listed in Table 1. The surface tension coefficient depends on the temperature, and thus induces a specific melting pool shape through Marangoni effects. The dependency of the surface tension with respect to the temperature depends on the composition of the material (typically density of sulfur), see, e.g., [9, 17, 18]. Numerical results are presented when this density is 150 [ppm] [16]. The enthalpy-temperature relation $\mathcal{T}=\beta(\mathcal{H})$ is taken as in [12].

Table 1: Material properties for Böhler S705 steel.

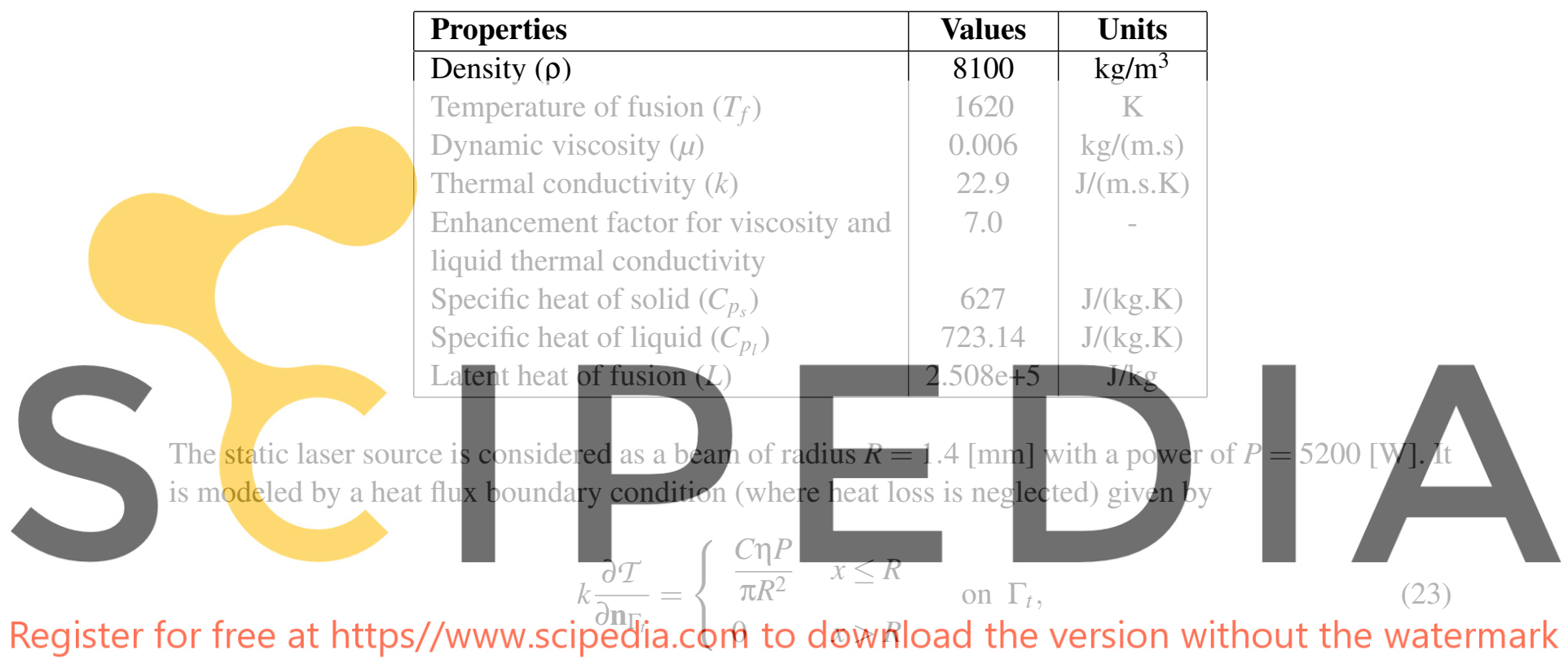

where $x$ denotes the horizontal distance, $\eta=0.13[-]$ is the metal absorption coefficient and $C=0.2$ is a constant to account for the pseudo-2D approximation. Adiabatic boundary conditions are imposed on the remaining parts of the boundary. The initial temperature is the ambient temperature such that $f_{\ell} \equiv 0$ in $\Omega_{0}$ (no liquid region).

For this kind of problem the gravitational forces can be neglected compared to surface tension forces (small Bond number). The coefficients in (4) are taken as $\bar{\alpha}=100$ and $\varepsilon=10^{-3}$. We apply slip boundary conditions in the $x z$-planes (pseudo-2D), no-slip boundary conditions on $\partial \Lambda$ and the natural force condition on the free surface $\Gamma_{t}$. The initial condition for the velocity is $\mathbf{v} \equiv \mathbf{0}$.

Figure 4 illustrates the solution after $T=0.15$ [s], namely the temperature and the velocity fields. Numerical experiments show a strong coupling between the thermal aspects and the deformation of the free surface, as well as strong internal currents in the melt pool, which are due to the Marangoni effects.

In a second step, a mesh convergence analysis is undertaken. At the final time, the modeling of surface forces is studied through a comparison of the two computational methods for surface tension effects $[5,6]$. Figure 5 shows the convergence behavior of the approximation of the free surface for three 

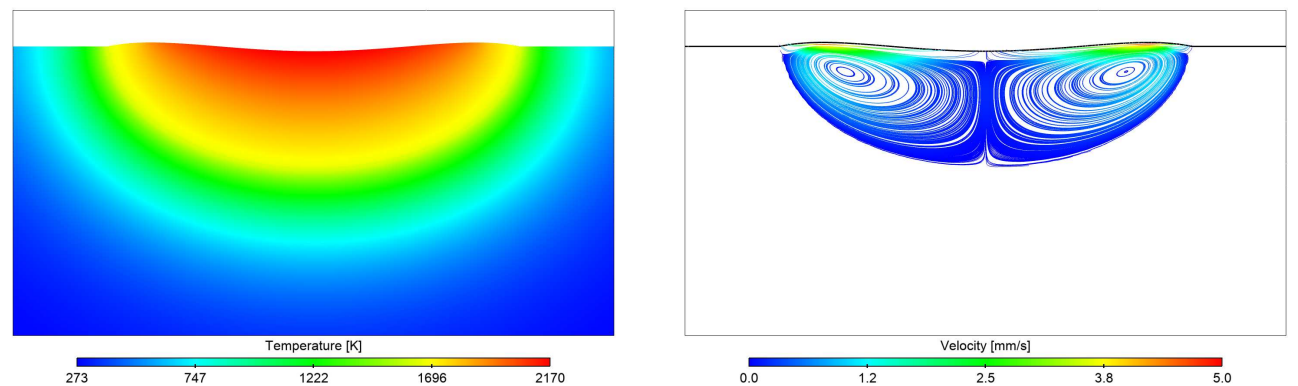

Figure 4: Pseudo-2D static laser melting (fine mesh). Snapshots at time $T=0.15[s]$ of the numerical approximation of the temperature $\mathcal{T}$ (left), and the velocity streamlines $\mathbf{v}$ (right).

mesh sizes, for the model with explicit calculation of the surface curvature [5] with $\varepsilon \simeq O(\sqrt{H})$ as the kernel smoothing parameter (1eft), and for the variational model [6] (middle). In both cases, we observe convergence of the approximation of the free surface towards a similar limit, although the model with an explicit calculation of the curvature shows some oscillations in the approximation of the free surface. This is due to the explicit calculation of the curvature, which is discontinuous at the transition between the liquid pool and the solid metal. Figure 5 (right) exhibits an appropriate first-order convergence rate for the error on the free surface deformation (with respect to the numerical solution computed on a very fine mesh).
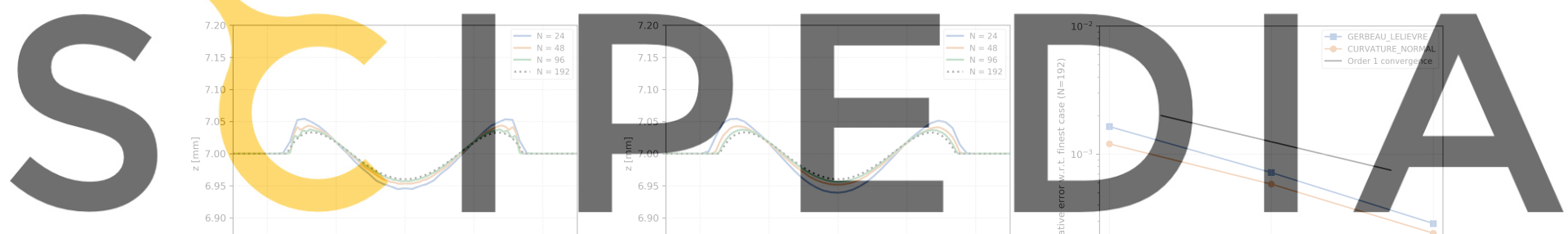

Register for free at https//www.scipedia.com to download the version without the watermark

Figure 5: Pseudo-2D static laser melting. Convergence analysis for the error on the approximation of the free surface deformation. Left: model with explicit calculation of the surface curvature ('curvature_normal') [5]; Middle: variational model ('gerbeau_lelievre') [6]; Right: first order error convergence rate for both methods.

\section{Acknowledgments}

The authors thank B. Meylan, K. Wassmer, P. Hoffman (EMPA), E. Boillat (EPFL), I. Calderon and J.-C. Prélaz (Unitechnologies SA) for fruitful discussions. All the computations were performed using the software cfsFlow++ developed by EPFL and Ycoor Systems SA.

\section{REFERENCES}

[1] A. Temmler, E. Willenborg, K. Wissenbach, Laser polishing, Proceedings of SPIE - The International Society for Optical Engineering 8243 (2012) 19-.

[2] E. V. Bordatchev, A. M. K. Hafiz, O. R. Tutunea-Fatan, Performance of laser polishing in finishing 
of metallic surfaces, Int. J. Adv. Manuf. Technol. 73 (1-4) (2014) 35-52.

[3] A. Paolini, S. Kollmannsberger, E. Rank, Additive manufacturing in construction: A review on processes, applications, and digital planning methods, Additive Manufacturing 30 (2019) 100894.

[4] B. Wucher, L. Arbaoui, Multiscale modeling of the sintering process of printed nanoinks, Computers and Mathematics with Applications 78 (7) (2019) 2325 - 2337, simulation for Additive Manufacturing.

[5] A. Caboussat, A numerical method for the simulation of free surface flows with surface tension, Computers and Fluids 35 (10) (2006) 1205-1216.

[6] J.-F. Gerbeau, T. Lelièvre, Generalized Navier boundary condition and geometric conservation law for surface tension, Comput. Methods Appl. Mech. Engrg. 198 (2009) 644-656.

[7] V. Maronnier, M. Picasso, J. Rappaz, Numerical simulation of three dimensional free surface flows, Int. J. Num. Meth. Fluids 42 (7) (2003) 697-716.

[8] M. Rappaz, M. Bellet, M. Deville, Modeling in Materials Science and Engineering, Springer Series in Computational Mathematics, Springer Verlag, Berlin Heidelberg, 2003.

[9] G. P. Sasmal, J. I. Hochstein, Marangoni convection with a curved and deforming free surface in a cavity, Journal of Fluids Engineering 116 (3) (1994) 577-582.

[10] D. Kothe, M. M. Francois, J. M. Sicilian, Modeling of thermocapillary forces within a volume tracking algorithm, Modeling of Casting, Welding and Advanced Solidification Processes - XI 2

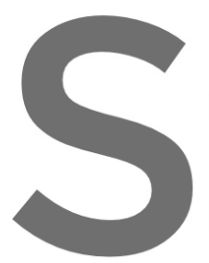
(2006) 935-942.

[11] M. Paolini, G. Sacc International Journal

[12] Y. Safa, M. Flueck hydrodynamic effects
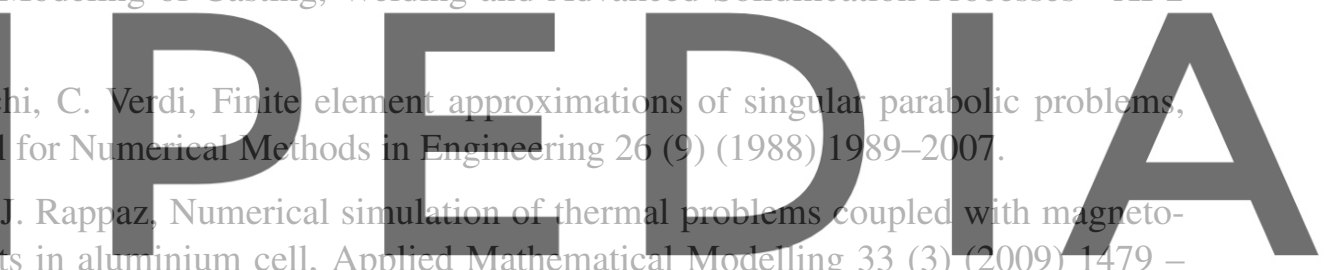
1492

Register for free at https//www.scipedia.com to d ownload the version without the watermark ing by adaptive Eulerian grid subdivision, Math. Comput. Modelling 55 (2012) 490-504.

[14] R. Franke, Scattered data interpolation: Test of some methods, Math. Comput. 38 (1982) 181-200.

[15] D. Arnold, F. Brezzi, M. Fortin, A stable finite element for the Stokes equations, Calcolo 21 (4) (1984) 337-344.

[16] A. Caboussat, J. Hess, A. Masserey, M. Picasso, A numerical model for the simulation of shallow laser surface melting, in: Proceedings of the II International Conference on Simulation for Additive Manufacturing Sim-AM 2019, 2019, pp. 286-296.

[17] W. Pitscheneder, T. Debroy, K. Mundra, R. Ebner, Role of sulfur and processing variables on the temporal evolution of weld pool geometry during multikilowatt laser beam welding of steels, Welding research 75 (1996) 71-80.

[18] P. Sahoo, T. Debroy, M. J. McNallan, Surface tension of binary metal - surface active solute systems under conditions relevant to welding metallurgy, Metallurgical Transactions B 19 (3) (1988) 483491. 